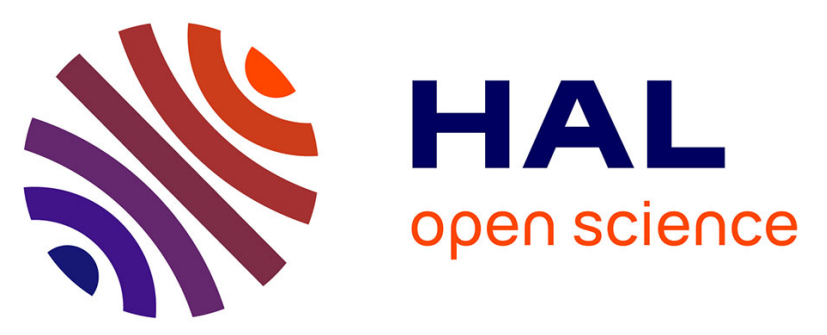

\title{
DATATION PAR TRACES DE FISSION ET ÉTUDE DE PROVENANCE D'ARTEFACTS EN OBSIDIENNE DES SITES ARCHÉOLOGIQUES DE LA TOLITA (Equateur) ET INGUAPI (Colombie)
}

Oliver Dorighel, Gérard Poupeau, Jean-François Bouchard, Erika Labrin

\section{To cite this version:}

Oliver Dorighel, Gérard Poupeau, Jean-François Bouchard, Erika Labrin. DATATION PAR TRACES DE FISSION ET ÉTUDE DE PROVENANCE D'ARTEFACTS EN OBSIDIENNE DES SITES ARCHÉOLOGIQUES DE LA TOLITA (Equateur) ET INGUAPI (Colombie). Bulletin de la S. P. F. T., 1994, 2, pp.133-144. 10.3406/bspf.1994.9726 . hal-02891877

\section{HAL Id: hal-02891877 https://hal.science/hal-02891877}

Submitted on 8 Jul 2020

HAL is a multi-disciplinary open access archive for the deposit and dissemination of scientific research documents, whether they are published or not. The documents may come from teaching and research institutions in France or abroad, or from public or private research centers.
L'archive ouverte pluridisciplinaire HAL, est destinée au dépôt et à la diffusion de documents scientifiques de niveau recherche, publiés ou non, émanant des établissements d'enseignement et de recherche français ou étrangers, des laboratoires publics ou privés. 


\title{
Datation par traces de fission et étude de provenance d'artefacts en obsidienne des sites archéologiques de la Tolita (Equateur) et Inguapi (Colombie)
}

\author{
Olivier Dorighel, Gérard Poupeau, Jean-François Bouchard, Erika Labrin
}

\section{Résumé}

RÉSUMÉ Nous avons daté par la méthode des traces de fission de l'Uranium onze artefacts en obsidienne provenant de deux sites côtiers : La Tolita (Nord-Équateur, 8 artefacts) et Inguapi (Sud-Colombie, 3 artefacts). Quatre groupes d'âges se dégagent de cette analyse, respectivement vers 0,25-0,30 Ma (six échantillons de La Tolita, un d'Inguapi), 0,42 Ma (La Tolita), 1,65 Ma (La Tolita) et 2,59 Ma (Inguapi). Les implications de la présence de trois de ces groupes d'âges dans les obsidiennes de la Sierra équatorienne et colombienne sont discutées en termes d'origine pour la matière première de ces artefacts.

\section{Abstract}

ABSTRACT Eleven obsidian artefacts from two coastal sites, La Tolita (North Ecuador, 8 artefacts) and Inguapi (South Colombia, 3 artefacts) were dated by fission tracks. Three plateau-age groups are revealed, respectively around $0.25-0.30 \mathrm{My}$ (six artefacts from La Tolita, one from Inguapi), $0.42 \mathrm{My}$ (La Tolita), 1.65 My (La Tolita) and 2.59 My (Inguapi). The results are discussed as regards the origin of these materials, taking into account that three of the age groups are also represented in the obsidians of the Sierra of Colombia and Ecuador.

\section{Citer ce document / Cite this document :}

Dorighel Olivier, Poupeau Gérard, Bouchard Jean-François, Labrin Erika. Datation par traces de fission et étude de provenance d'artefacts en obsidienne des sites archéologiques de la Tolita (Equateur) et Inguapi (Colombie). In: Bulletin de la Société préhistorique française, tome $91, \mathrm{n}^{\circ} 2,1994$. pp. 133-144;

doi : https://doi.org/10.3406/bspf.1994.9726

https://www.persee.fr/doc/bspf_0249-7638_1994_num_91_2_9726

Fichier pdf généré le 13/04/2018 


\title{
DATATION PAR TRACES DE FISSION ET ÉTUDE DE PROVENANCE D'ARTEFACTS EN OBSIDIENNE DES SITES ARCHÉOLOGIQUES DE LA TOLITA (Équateur) ET INGUAPI (Colombie)
}

\author{
Olivier DORIGHEL, Gérard POUPEAU, Jean-François BOUCHARD et Erika LABRIN
}

\section{RÉSUMÉ}

Nous avons daté par la méthode des traces de fission de l'Uranium onze artefacts en obsidienne provenant de deux sites côtiers : La Tolita (Nord-Équateur, 8 artefacts) et Inguapi (Sud-Colombie, 3 artefacts). Quatre groupes d'âges se dégagent de cette analyse, respectivement vers $0,25-0,30 \mathrm{Ma}$ (six échantillons de La Tolita, un d'Inguapi), 0,42 Ma (La Tolita), 1,65 Ma (La Tolita) et 2,59 Ma (Inguapi). Les implications de la présence de trois de ces groupes d'âges dans les obsidiennes de la Sierra équatorienne et colombienne sont discutées en termes d'origine pour la matière première de ces artefacts.

\section{ABSTRACT}

Eleven obsidian artefacts from two coastal sites, La Tolita (North Ecuador, 8 artefacts) and Inguapi (South Colombia, 3 artefacts) were dated by fission tracks. Three plateau-age groups are revealed, respectively around $0.25-0.30 \mathrm{My}$ (six artefacts from La Tolita, one from Inguapi), 0.42 My (La Tolita), 1.65 My (La Tolita) and 2.59 My (Inguapi). The results are discussed as regards the origin of these materials, taking into account that three of the age groups are also represented in the obsidians of the Sierra of Colombia and Ecuador.

\section{INTRODUCTION}

II y a pratiquement trente ans naissait la méthode de datation par les Traces de Fission (TF) de I'Uranium (Price et Walker, 1963). Applicable à l'ensemble des temps géologiques, elle devait entrer rapidement dans le domaine de l'archéométrie (Fleischer et al., 1965 a). L'apport de la méthode des traces à l'archéolo- gie tient à sa capacité à dater des roches volcaniques récentes (Wagner, 1978 ; Poupeau, 1979). Elle peut ainsi participer à la datation de sols brûlés (Guo Shilun et al., 1980, 1990), de niveaux-repères constitués de cendres volcaniques (Fleischer et al., 1965 b ; Gleadow, 1980 ; Hurford et Watkins, 1987, etc.), comme à la caractérisation d'artefacts en obsidienne (Fleischer et al.,1965 a ; Bigazzi et al., 1990, etc.).

En 1973, Suzuki montrait que deux paramètres fournis par la méthode des traces : âge et teneur en Uranium, permettaient de caractériser de manière univoque les artefacts préhistoriques en obsidienne de la région de Tokyo et de les rapporter à leurs gisements géologiques. Le même auteur avait aussi prouvé que lorsqu'un fragment d'obsidienne a été chauffé artificiellement, comme constituant d'une poterie, son âge TF était celui du dernier réchauffement de celle-ci audelà de $500{ }^{\circ} \mathrm{C}$ (Watanabe et Suzuki, 1969). Miller et Wagner (1981), de leur côté, démontraient que dans le cas d'un chauffage modéré, comme dans un foyer, il pouvait être possible d'atteindre à la fois l'âge de l'obsidienne et celui de son utilisation par l'homme.

Dans les Andes de Colombie et d'Équateur, l'approche TF a été appliquée à plusieurs sites archéologiques et gîtes géologiques dans la perspective d'études de datation de sites, de circulation et de provenance de l'obsidienne (Miller et Wagner, 1981 ; Arias et al., 1986 ; Bigazzi et al., 1992). L'intérêt de l'utilisation conjointe de la méthode des traces et de l'étude de la composition chimique des obsidiennes a été montré par Bigazzi et al. (1986, 1992). Nous présentons ici les premiers résultats d'un programme de caractérisation géochronologique et géochimique d'artefacts en obsidienne de la région côtière Nord-Équateur/Sud-Colombie. II s'agit de la datation par TF d'échantillons prélevés par l'un d'entre nous (J.-F. B.) dans les sites archéologiques de La Tolita (Équateur) et d'Inguapi (Colombie).

\section{LA DATATION PAR TRACES DE FISSION}

\section{- Principes généraux}

L'aptitude de l'Uranium à fissionner spontanément est à la base de la méthode de datation par TF. La scission du noyau de l'atome d'Uranium $238\left({ }^{238} \mathrm{U}\right)$ en deux noyaux-fils libère une énergie considérable, presque entièrement convertie en énergie cinétique, qui va entraîner le recul de ces noyaux, ou fragments de fission, selon des directions opposées. II en résulte, dans un minéral ou un verre naturels, la formation d'un défaut initial, ou trace latente, invisible au microscope : si la longueur d'une de ces traces peut atteindre plus de 20 microns dans certains matériels (micas), son diamètre, par contre, ne dépasse pas un centième de micron. Par contre, ces défauts peuvent être visualisés grâce à une attaque chimique, qui agrandit leur diamètre jusque vers au moins 0,5 micron. Ces traces "révélées", que va utiliser le géochronologiste, sont celles qui seront recoupées par une surface de polissage du matériel à étudier. Leur morphologie varie selon la différence entre la vitesse de dissolution le long d'une trace latente et celle qui caractérise le matériel sain. C'est ainsi que dans un verre volcanique, les traces révélées présentent une allure circulaire à elliptique (fig. 1).

Dans un échantillon (minéral, verre volcanique) plio-quaternaire, le nombre de traces de fission spontanée, ou traces fossiles, est proportionnel au temps depuis lequel ces traces sont enregistrées, ainsi qu'à la teneur en Uranium. Afin de tenir compte de cette dernière, on irradie l'échantillon en réacteur nucléaire pour faire fissionner (par capture de 

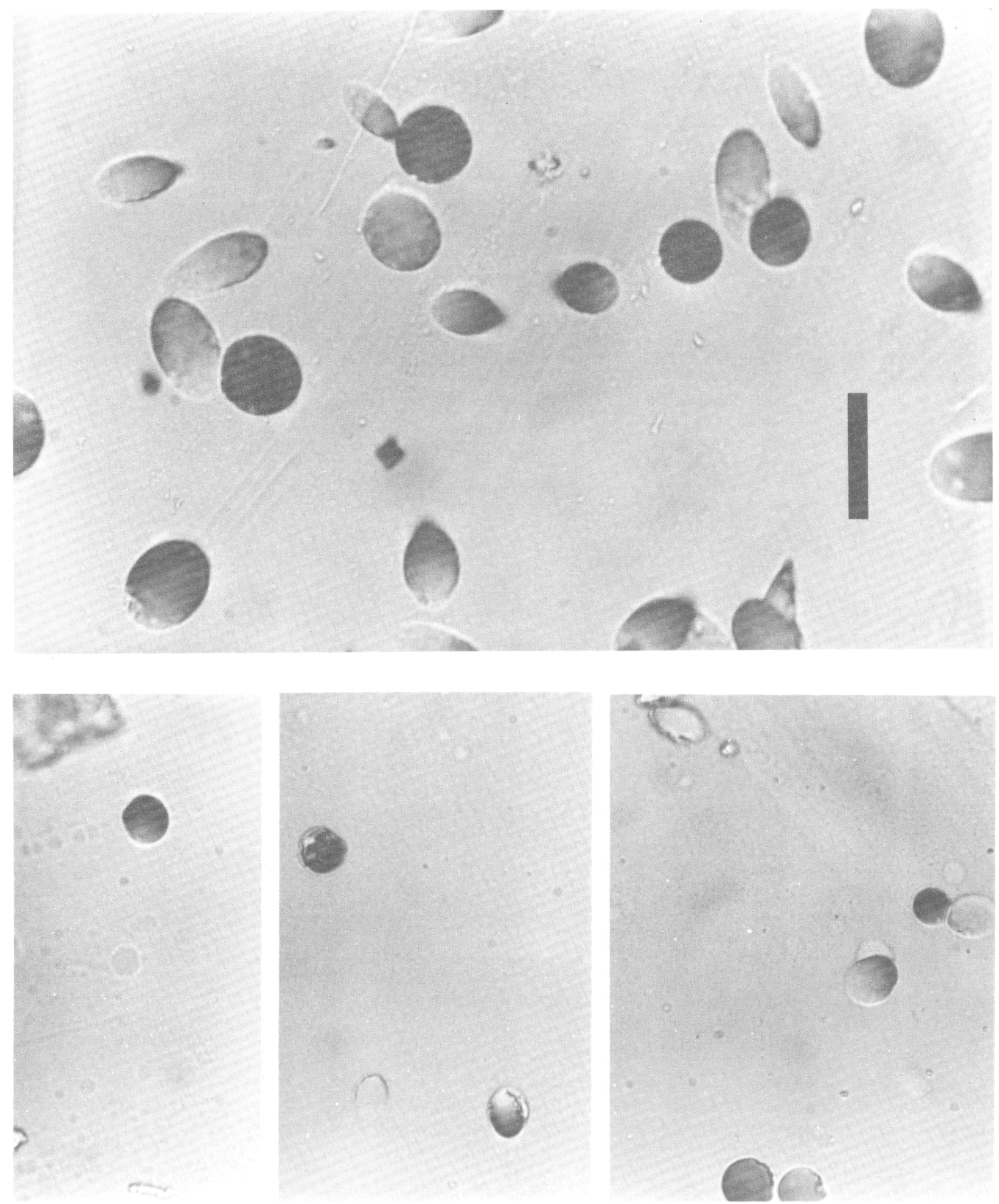

Fig. 1 - Traces de fission dans un verre naturel, une obsidienne d'Inguapi. Les traces présentent une forme circulaire à elliptique, en fonction de leur orientation par rapport à la surface d'observation. La barre d'échelle vaut 20 microns. On observe que les traces de fission fossiles (photos du bas) sont plus petites que les traces de fission induite en réacteur nucléaire (photo du haut). Dans cet échantillon, la densité de traces induites est de $3,8 \times 10^{5}$ traces $/ \mathrm{cm}^{2}$ (tabl. 1, échantillon Inguapi I3). Le diamètre moyen $\left(D_{i}\right)$ des traces induites est de 12,47 microns, tandis que celui $\left(D_{f}\right)$ des traces fossiles $n$ 'est que de 9,48 microns (fig. 3).

neutrons thermiques) un autre isotope de l'Uranium, I'Uranium 235 $\left({ }^{235} \mathrm{U}\right)$, dont on peut de la même façon que précédemment, étudier les traces de fission induite. Comme dans la nature le rapport ${ }^{235} \mathrm{U} /{ }^{238} \mathrm{U}$ est constant et connu, l'équation d'âge de la datation par TF pour les temps plio-quaternaires se réduit à l'expression :

$$
t=A\left(\rho_{f} / \rho_{j}\right) \Phi
$$

où t est l'âge de l'échantillon (Ma), A un terme regroupant plusieurs constantes, et $\rho_{\mathrm{f}}$ et $\rho_{\mathrm{i}}$ respectivement les densités (nombre/cm²) de traces de ${ }^{238} \mathrm{U}$ et ${ }^{235} \mathrm{U}$ du matériau à dater. Le terme $\Phi$ est la fluence de neutrons thermiques utilisée ; la fluence est calculée à partir de la densité de traces de fission induite de ${ }^{235} \mathrm{U}$ me- 
surée dans une pastille de verre (ou un détecteur associé) contenant une quantité connue d'Uranium et irradiée conjointement à l'échantillon à dater. Cette procédure implique une calibration à partir d'échantillons d'âges connus (Poupeau et al., 1994). La détermination de ces trois densités de traces est réalisée en microscopie optique. Pour ce faire, on effectue un balayage de l'échantillon et du moniteur de verre (ou de son détecteur de traces associé), en dénombrant les traces rencontrées pour chaque champ visuel.

\section{- La datation des obsidiennes}

Cette datation est réalisée par la méthode dite de la soustraction (Gleadow, 1981). La teneur en Uranium dans un verre volcanique étant extrêmement homogène, on procède de la façon suivante : deux fragments, d'une dimension d'environ $4 \times 4 \times 3 \mathrm{~mm}$ chacun, sont prélevés sur l'échantillon à dater. Dans l'un d'entre eux sera mesurée la densité de traces fossiles $\rho_{f}$, généralement très faible, inférieure à $10^{4}$, voire $10^{3}$ traces $/ \mathrm{cm}^{2}$. L'autre fragment sera exposé à une dose de neutrons thermiques suffisante pour produire une densité de traces induites de l'ordre de $10^{5}$ traces $/ \mathrm{cm}^{2}$. La densité de traces de fission induite dans ce fragment sera calculée selon:

$$
\rho_{\mathrm{i}}=\rho^{\star}-\rho_{\mathrm{f}}
$$

où $\rho^{\star}$ est la densité mesurée après irradiation.

Après l'irradiation, on inclut dans une même pastille d'araldite à la fois le fragment non irradié et celui qui est passé en réacteur nucléaire. $\mathrm{Ce}$ montage permet de traiter les deux aliquotes d'une manière identique vis-à-vis du polissage et de l'attaque chimique qu'ils doivent subir pour la révélation des traces.

Dans les datations par traces de fission, la teneur en Uranium n'est pas formellement calculée : elle intervient par le paramètre $\rho_{i}$. II est cependant important de la connaitre si l'on s'intéresse à la caractérisation d'une obsidienne. On irradie alors à la fois un moniteur de verre (de teneur en $U$ connue) et l'échantillon à dater, chacun poli et associé à un "détecteur externe", feuille de plastique ou de mica muscovite (très pauvre en Uranium). La comparaison des traces de fission induites des détecteurs asso- ciés respectivement au moniteur et à l'échantillon permet de déterminer la teneur en Uranium de ce dernier avec une précision de l'ordre de $\pm 5 \%$ (Fleischer et al., 1975).

\section{Ages apparents, âges corrigés}

On appelle "âge apparent" l'âge calculé selon l'équation (1). Cet âge n'a de signification précise que si les traces fossiles n'ont subi aucune modification depuis leur enregistrement dans le matériel à dater. Or on sait que dans les verres volcaniques existe un processus de vieillissement ("aging") des traces latentes, dont la résultante est un raccourcissement des traces fossiles révélées (fig. 2). II en résulte un rajeunissement de l'âge TF, qui dès lors n'est plus qu'un âge minimum de début d'enregistrement des traces. II est donc indispensable de vérifier si un âge apparent TF a été affecté par ce phénomène, et de savoir le "corriger" de cet effet de "cicatrisation" partielle des traces latentes.

Les traces de fission fossiles et induites doivent présenter, en l'absence d'effet perturbateur, les mêmes dimensions, en raison de la similitude énergétique des fissions spontanées de ${ }^{238} \mathrm{U}$ et induites de ${ }^{235} \mathrm{U}$, comme vérifié expérimentalement par Bigazzi et al. (1991). Toute datation par TF d'un verre (naturel ou fabriqué) doit donc être accompagnée d'une caractérisation de la conservation des longueurs révélables des traces fossiles. Dans le cas des verres, où les traces présentent une allure arrondie à ellipsoïdale, on utilise la mesure du plus grand diamètre de ces figures de corrosion. Dans le cas où les distributions de diamètres de traces fossiles et induites ne sont pas superposables, on doit utiliser une procédure de correction permettant d'atteindre un âge significatif.

La procédure des "âges-plateaux" (Storzer et Poupeau, 1973) est actuellement la plus utilisée. Cette technique repose sur la propriété des traces de "s'effacer" progressivement avec la température : on constate en effet qu'à la suite d'un traitement thermique approprié, les traces d'une obsidienne, pour des conditions de révélation identiques, peuvent voir leur taille, et donc leur densité, diminuer. Après un chauffage d'une heure à $500^{\circ} \mathrm{C}$, il n'est plus possible de révéler de traces dans l'obsidienne. Dans la procédure des âges-plateaux, on détermine tout d'abord un âge apparent, puis les diamètres moyens $D_{f}$ et $D_{i}$ des traces fossiles et induites. Si le rapport $D_{f} / D_{i}<1$, on soumet l'échantillon à un traitement thermique approprié, en général 1 à 2 heures à une température légèrement supérieure à $200^{\circ} \mathrm{C}$, et on mesure à nouveau le rapport $D_{f} / D_{i}$ (fig. 2). $C e$ chauffage affectant principalement la population de traces induites produites en réacteur et donc non encore atteinte par le processus d'aging, le rapport $D_{f} / D_{i}$ doit alors se rapprocher de 1 (fig. 3). Selon les échantillons, il faut une ou plusieurs étapes de traitement thermique à des températures croissantes (plateau-isochrone, Storzer et Poupeau, 1973), ou à température constante mais pour des temps plus longs (plateau-isotherme, pour les verres hydratés, Poupeau, 1982), pour ramener le rapport $D_{f} / D_{i}$ à l'unité (fig. 2). A ce moment, les deux populations de traces, fossiles et induites, ont été ramenées à un même taux d'effacement. Leurs distributions de diamètres de traces sont à nouveau identiques (comme si aucune de ces deux populations n'avait été affectée d'effacement partiel) et l'âge TF associé correspond alors à un événement bien déterminé.

\section{Signification d'un âge TF}

Lorsqu'aucun processus d'aging n'affecte les traces d'une obsidienne ou lorsqu'un âge-plateau a été obtenu, l'événement daté est celui du dernier refroidissement au-dessous d'environ $500{ }^{\circ} \mathrm{C}$. En général ce sera l'âge de l'éruption volcanique. II peut toutefois arriver qu'un artefact d'obsidienne ait été passé au feu (intentionnellement ou non) à l'époque de son utilisation. Dans ce cas, les traces préexistantes ont pu être partiellement effacées, ce qui affecte l'âge apparent, mais non l'âge-plateau de cet artefact. Par contre, si ce chauffage a été suffisant $\left(>500{ }^{\circ} \mathrm{C}\right)$ pour "effacer" toutes les traces fossiles, l'âge obtenu sera celui du réchauffement le plus récent. Dans certaines circonstances favorables: effacement presque total des traces pré-existantes par passage au feu, on peut éventuellement dater à la fois, sur le même fragment d'obsidienne, l'âge de l'éruption et celui de son réchauffement ultérieur (Miller et Wagner, 1981). 


\section{LES SITES ARCHÉOLOGIQUES DE LA TOLITA ET D'INGUAPI}

\section{- Contexte archéologique}

Les sites archéologiques d'Inguapi et de La Tolita appartiennent à la région du littoral pacifique nordéquatorial, couvrant les côtes du Sud de la Colombie et du Nord de l'Équateur (fig. 4). Pendant l'époque préhispanique, cette région connut un développement culturel très important entre 700-600 BC et environ $300 \mathrm{AD}$. La dernière partie de cette période (entre $300 \mathrm{BC}$ et $300 \mathrm{AD}$ ) correspond à la phase culturelle "La Tolita-Tumaco", qui constitue l'un des Développements Régionaux de la périodi-
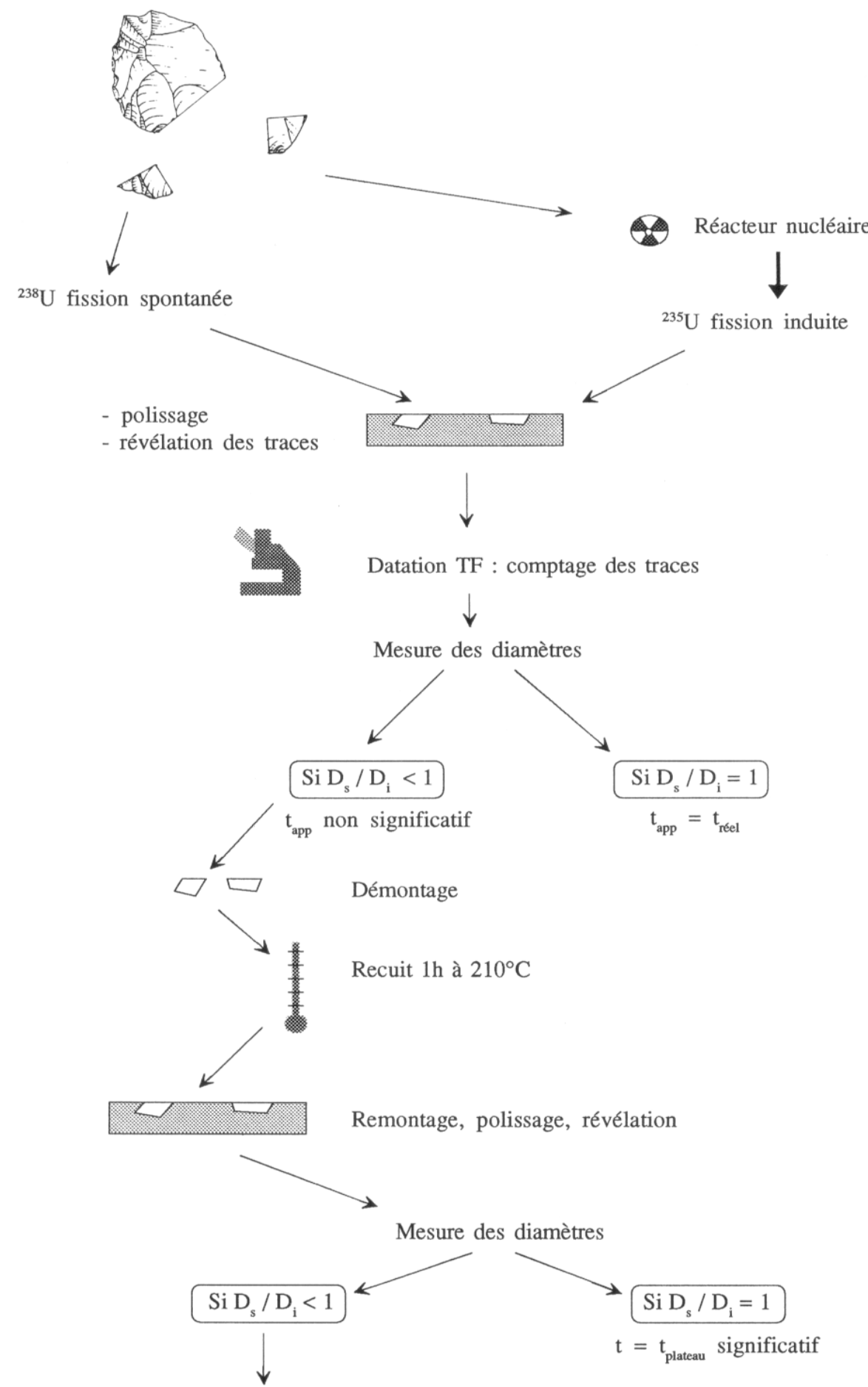

Nouveau recuit : $2 \mathrm{~h}$ à $215^{\circ} \mathrm{C}$

Fig. 2 - Principe de la datation des artefacts d'obsidienne par àges-plateaux. Lorsque les traces fossiles présentent un raccourcissement dû aux conditions d'environnement de leur gisement géologique ou archéologique (température, hydratation), les traces fossiles peuvent présenter un raccourcissement caractérisé par une valeur du rapport $D_{f} / D_{i}<1$. On retrouve la condition d'applicabilité de l'équation de datation par TF, soit $D_{f} / D_{i}=1$ en pratiquant un ou plusieurs recuits.

fication chrono-culturelle de l'aire andine équatoriale. Auparavant, comme les plus récentes recherches viennent de l'établir, cette région fut occupée par une phase antérieure entre environ $700-600 \mathrm{BC}$ et $300 \mathrm{BC}$. Ces premiers occupants, agriculteurs céramistes, développèrent alors des traditions culturelles qui évoluèrent peu à peu pour donner naissance vers $300 \mathrm{BC}$ à la phase La Tolita-Tumaco, comme l'atteste entre autres la filiation établie entre les divers corpus constitués pour la classification céramique (Bouchard, 1984, 1986, 1989 a, b ; Valdez, 1986, 1987).

Inguapi, près de Tumaco en Colombie et La Tolita Cancha en Équateur, sont deux sites archéologiques qui ont livré des niveaux culturels attribuables à ces deux grandes périodes d'occupation. A La Tolita, il a été possible d'effectuer des ramassages de surface dans les déblais de fouilles clandestines en vue d'obtenir un matériel archéologique complémentaire. C'est de tels ramassages que proviennent les échantillons d'obsidienne analysés. En revanche, ceux d'Inguapi proviennent bien, pour leur part, de niveaux en place. Dans les deux cas, les pièces sont attribuables sans doute possible à cette période d'occupation préhispanique (Bouchard, 1984, 1986, 1989 a, b).

Le très grand nombre de sépultures découvertes (principalement par les fouilleurs clandestins) permet de penser que La Tolita fut une nécropole où étaient rassemblés après leur mort les plus importants personnages de cette civilisation. L'île joua aussi un rôle de métropole culturelle et sans doute politique, colonisant les régions voisines le long des côtes mais aussi le long des cours d'eau donnant accès à la plaine alluviale. Divers sites en bordure de cours d'eau ont ainsi été découverts, aussi bien en Colombie qu'en Équateur (Bouchard, 1984, 1986, 1989 a, b ; Deboër et Tolstoy, 1989).

Le site d'Inguapi constitue un exemple de ces sites où se reconnaît l'influence de la culture La Tolita-Tumaco. Il est situé en bordure d'un chenal de marée (cours d'eau se remplissant uniquement à marée haute) et témoigne de deux occupations anciennes (Inguapi 1 et Inguapi 2) ainsi que d'une occupation beaucoup plus tardive. Les vestiges d'obsidienne recueillis sur ce site ne relèvent que des phases an- 

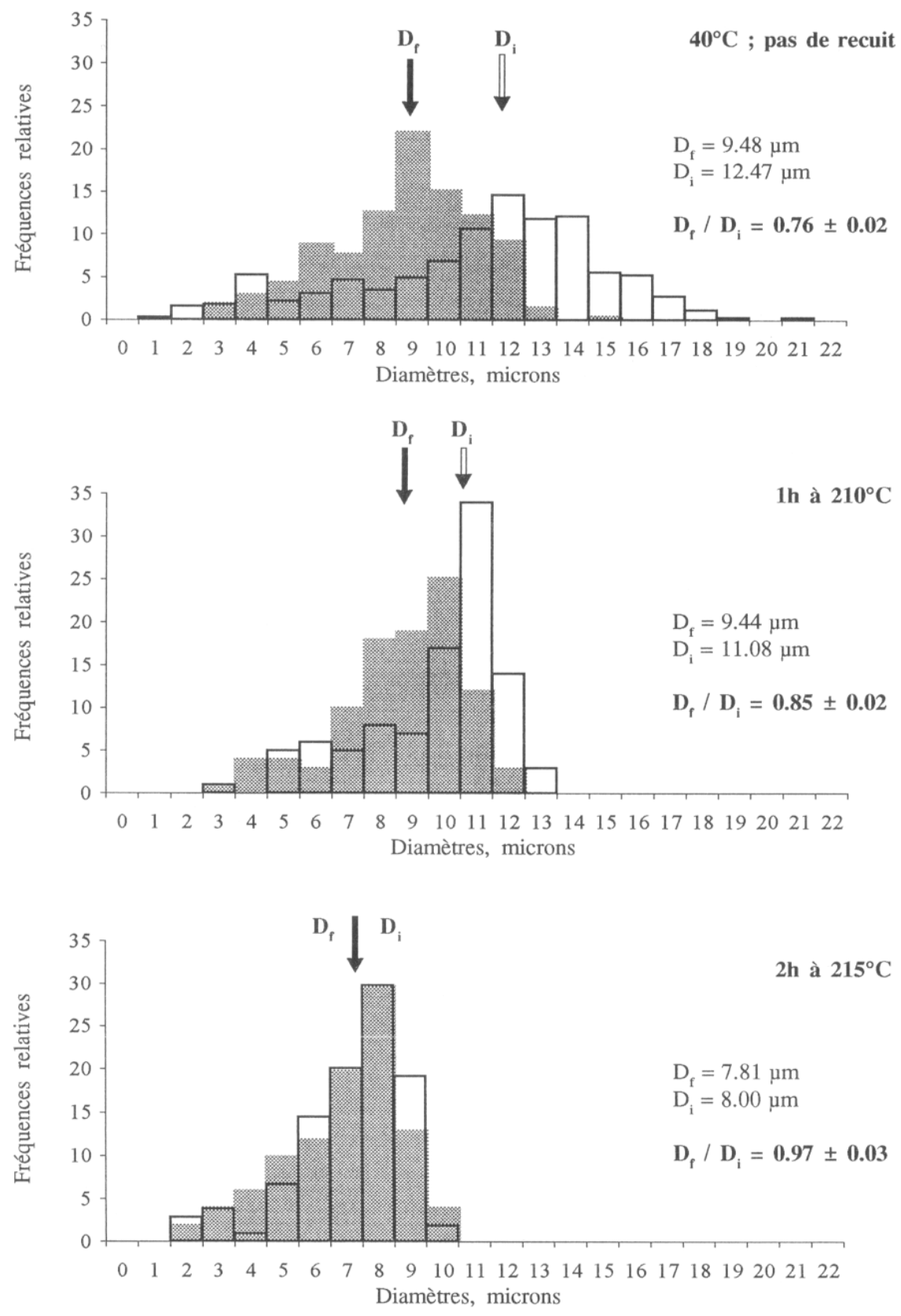

Fig. 3 - Distribution des diamètres de traces fossiles (en grisé) et induites avant et après traitement thermique, pour l'échantillon I3 du site d'Inguapi. On remarque (histogrammes du haut) que les traces fossiles sont affeccuit à $210^{\circ} \mathrm{C}$ (fig. centrate) modifie surtout les traces induites, qui restent cependant en moyenne plus longues que les fossiles. Après un second recuit à $215^{\circ} \mathrm{C}$ (bas), les deux populations de traces présentent la même distribution de diamètres, condition d'application de l'équation d'áge.

(Dans les traces elliptiques - voir fig. 1 - le paramètre mesuré est le plus grand diamètre, c'est-à-dire celui qui est pris dans la direction d'allongement maximum).

ciennes Inguapi 1 ou 2. Le plus ancien niveau d'occupation possède encore divers traits technologiques attribuables à la période précédant les Développements Régionaux, alors que le second niveau, un peu plus récent, ne possède déjà plus qu'une céramique à la gamme de décors très réduite par rapport à celle du niveau Inguapi 1 . Sur le plan de l'interprétation archéologique que l'on peut proposer, Inguapi 1 semble correspondre à l'arrivée des "colons" appartenant à une tradition culturelle originaire de la région de La Tolita ;
Inguapi 2 appartiendrait à la phase "classique" de la civilisation La Tolita-Tumaco.

\section{- La question de la matière première}

La présence d'objets archéologiques en obsidienne, dans une région côtière où cette matière n'est pas naturellement présente, implique qu'il s'agit bien d'un matériau allochtone, ce qui a donné lieu à plusieurs interprétations archéologiques. Des origines lointaines furent d'abord évoquées, selon une hypothèse dite diffusioniste qui suggérait un peuplement venant de Méso-Amérique (Uhle, 1927 a, 1927 b ; Ferdon et Corbett, 1941 ; Cubillos, 1955 ; Reichel et Dolmatoff, 1965, 1987 ; Meggers, 1966).

L'obsidienne fait partie des matériaux fréquemment employés par les civilisations méso-américaines. Sa présence en Équateur, au lieu d'être attribuée à des sources andines, fut souvent considérée comme l'un des indices démontrant l'arrivée de migrations sur les côtes de l'Équateur à partir de la Méso-Amérique. Ce n'est qu'après 1970 que fut pleinement considérée l'hypothèse pourtant la plus logique selon laquelle l'obsidienne découverte sur les sites côtiers pouvait aussi bien provenir des gisements de la Sierra andine. Concordant mieux avec les réalités de la navigation qui rend difficile le voyage par mer depuis la MésoAmérique jusqu'à la côte Nord-équatoriale (Alcina-Franch et al., 1987), cette hypothèse présuppose toutefois un système d'exploitation de cette matière première fondée sur des expéditions entre la côte et la Sierra ou sur un trafic interrégional que l'état actuel des connaissances archéologiques ne nous permet pas encore de connaître (routes, ethnies concernées, produits, etc.).

La méthode de datation par TF appliquée à l'obsidienne découverte sur ces côtes constitue donc potentiellement un apport considérable à l'avance des connaissances en archéologie pour cette région des Andes équatoriales. L'identification des gisements d'obsidienne peut confirmer l'origine andine de cette matière première découverte sur la côte, permettant ainsi de mieux comprendre la manière dont les populations préhispaniques développèrent leur économie. En outre, si les résultats futurs continuent d'apporter l'évidence d'une telle origine, nous serions alors en mesure d'interpréter la présence d'obsidienne sur la côte non plus comme l'indice erroné de contacts lointains, mais comme celui, plus satisfaisant, de relations (dont le détail demeure à préciser) entre la côte et la Sierra au sein d'un développement culturel propre aux groupes de l'aire andine Nord-équatoriale. La méthode des traces de fission apporterait donc ainsi une évi- 

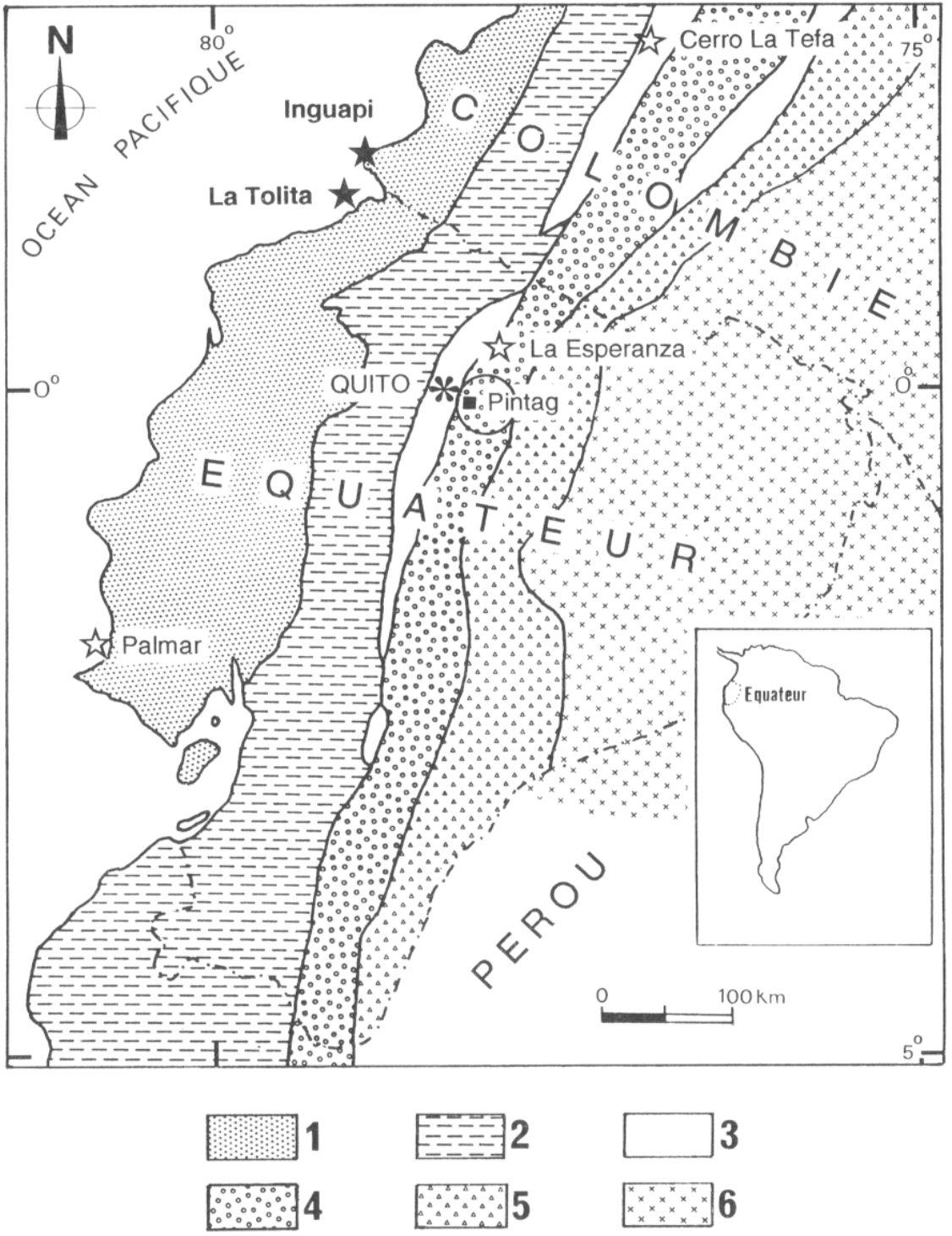

Fig. 4 - Carte géologique schématique de l'Équateur et du Sud de la Colombie, montrant la localisation des sites mentionnés dans le texte (adaptée de Bigazzi et al., 1992). 1 , région côtière ; 2 , Cordillère Occidentale 3 , dépression intra-cordillères ; 4, Cordillère Royale (Équateur) ou Centrale (Colombie) ; 5 , zone sub-andine ; 3, dépression intra-cordillères ; 4, Cordillère Royale (Equateur) ou Centrale (Colombie) ; 5 , zone sub-andine
6 , plate-forme amazonienne. Les étoiles situent les sites archéologiques mentionnés dans le texte : symboles pleins pour les deux sites d'où proviennent les artefacts datés dans ce travail ; symboles ouverts pour les autres sites. La zone cerclée où se trouve la ville de Pintag est celle qui renferme les plus importants gisements d'obsidienne d'Équateur, où ont été échantillonnés les obsidiennes datées par Bigazzi et al. (1992).

dence supplémentaire de la capacité des populations anciennes de cette région à se procurer dans leurs propres milieux les matériaux nécessaires à leur développement technologique.

A l'Est de Quito, la région d'llalo a abrité de nombreux chasseurscueilleurs paléo-indiens qui dès 10000 à $9000 \mathrm{BP}$ exploitèrent l'obsidienne, abondante dans cette zone (Bell, 1965 ; Mayer-Oakes, 1986). D'après Salazar (1992), il semblerait qu'il faille distinguer deux phases principales de l'usage de l'obsidienne en Équateur. Présente dans les zone de montagnes dès $10000 \mathrm{BP}$, elle apparaîtrait sur les et 3 issus des recherches à InguapiTumaco. L'ensemble du matériel s'est avéré être datable par TF. Nous avons utilisé la technique de datation par soustraction, la correction des âges vis-à-vis de l'effacement partiel des traces de fission spontanée étant assurée par la mesure d'âgesplateaux.

Les irradiations ont été pratiquées dans le canal P1 du réacteur Orphée (Centre d'Études Nucléaires de Saclay). La dosimétrie neutronique a été assurée au moyen des verres étalons \# 962 du National Institute of Standards and Technology des États-Unis (Gaithersburg, Maryland), selon les procédures en vigueur dans notre laboratoire (Poupeau et al., 1993, 1994). La révélation des traces de fission dans les obsidiennes a été obtenue par l'acide fluorhydrique en dilution $20 \%$. Les attaques ont été effectuées sous une température de $20^{\circ} \mathrm{C}$ ou $40^{\circ} \mathrm{C}$, contrôlée à $\pm 1^{\circ} \mathrm{C}$. La durée de l'attaque, adaptée à chaque échantillon, variait de 90 à 270 secondes à $20^{\circ} \mathrm{C}$, et de 180 à 240 secondes à $40^{\circ} \mathrm{C}$.

Les densités de traces ont été mesurées avec un microscope Leitz "Orthoplan" muni d'un objectif x 100 à sec, d'oculaires $\times 10$ et les mesures de diamètres effectuées dans les mêmes conditions, avec un microscope Zeiss-Jena "Jenavert" équipé d'une chambre claire et d'une table à digitaliser reliée à un microordinateur (tabl. 1).

Les résultats ont été reportés dans le tableau 1. Les densités de traces fossiles des obsidiennes étant très faibles, et donc les difficultés de distinction entre traces et inclusions ou autre défauts du verre se trouvant augmentées (Bigazzi et al., 1988 b), sept échantillons de La Tolita, LT2 à LT8, et l'échantillon d'Inguapi I3, ont été datés par deux observateurs. On constate que dans tous les cas les résultats obtenus sont concordants. L'augmentation apparente des densités de traces après recuit dans certains échantillons (comme LT2, LT3, etc.) résulte de conditions de révélation différentes avant et après traitement thermique. Par ailleurs, on observe que les traces de fission fossiles ont, dans tous les échantillons, subi un certain effacement naturel, comme en témoignent les valeurs du rapport $D_{f} / D_{i}$, toutes significativement inférieures à 1 (voir la fig. 3 pour l'échantillon 13). Là encore, lorsque ce rapport est déter-
Nous avons disposé pour ce travail de 11 échantillons, 8 provenant de la fouille Cancha H9 de La Tolita

\section{PROCEEDURES
EXPÉRIMENTALES ET RÉSULTATS}


Tableau 1

Datations par traces de fission. Données analytiques

\begin{tabular}{|c|c|c|c|c|c|c|c|c|c|c|}
\hline Ech. & Obs. & Nf & $\rho f_{\left(t / \mathbf{c m}^{2}\right)}^{ \pm 1 \sigma}$ & $\mathrm{Ni}$ & $\rho i \underset{\left(t / \mathbf{c m}^{2}\right)}{ \pm} 1 \sigma$ & $\begin{array}{c}\Phi \pm 1 \sigma \\
\left(10 \exp 15 \mathrm{n} / \mathrm{cm}^{2}\right)\end{array}$ & $\begin{array}{c}\text { Recuit } \\
\text { temps } / \mathbf{T}^{\circ} \mathrm{C}\end{array}$ & Df/Di & $\begin{array}{l}\mathbf{t} \pm 1 \sigma \\
(\mathbf{M a})\end{array}$ & $\underset{(\mathbf{p p m})}{\mathrm{Cu} \pm 1 \sigma}$ \\
\hline \multicolumn{11}{|l|}{ La Tolita } \\
\hline \multirow[t]{2}{*}{ LT1 } & 1 & 120 & $860 \pm 80$ & 2519 & $189700 \pm 3800$ & $0,777 \pm 0,013$ & aucun & 0,89 & $0,21 \pm 0,02$ & $3,9 \pm 0,2$ \\
\hline & 1 & 81 & $610 \pm 70$ & 1744 & $131300 \pm 3100$ & $1,062 \pm 0,013$ & $1 \mathrm{~h}$ à $210^{\circ} \mathrm{C}$ & 1,04 & $0,30 \pm 0,03$ & \\
\hline \multirow[t]{4}{*}{ LT2 } & 1 & 77 & $580 \pm 70$ & 2021 & $152200 \pm 3400$ & $0,777 \pm 0,013$ & aucun & 0,80 & $0,18 \pm 0,02$ & $5,9 \pm 0,2$ \\
\hline & 2 & 107 & $650 \pm 60$ & 2244 & $169000 \pm 3600$ & $0,777 \pm 0,013$ & aucun & & $0,18 \pm 0,02$ & \\
\hline & 1 & 97 & $730 \pm 70$ & 1634 & $122800 \pm 3000$ & $0,777 \pm 0,013$ & $1 \mathrm{~h}$ à $210^{\circ} \mathrm{C}$ & 0,98 & $0,28 \pm 0,03$ & \\
\hline & 2 & 95 & $730 \pm 70$ & 1902 & $109900 \pm 2500$ & $0,777 \pm 0,013$ & $1 \mathrm{~h}$ à $210^{\circ} \mathrm{C}$ & & $0,31 \pm 0,03$ & \\
\hline \multirow[t]{4}{*}{ LT3 } & 1 & 60 & $450 \pm 60$ & 1535 & $115600 \pm 3000$ & $0,777 \pm 0,013$ & aucun & 0,82 & $0,18 \pm 0,02$ & $5,5 \pm 0,2$ \\
\hline & 2 & 63 & $425 \pm 50$ & 1655 & $118800 \pm 2900$ & $0,777 \pm 0,013$ & aucun & & $0,17 \pm 0,02$ & \\
\hline & 1 & 114 & $750 \pm 70$ & 1681 & $126400 \pm 3000$ & $0,777 \pm 0,013$ & $1 \mathrm{~h}$ à $210^{\circ} \mathrm{C}$ & 0,96 & $0,28 \pm 0,02$ & \\
\hline & 2 & 95 & $810 \pm 80$ & 2127 & $133200 \pm 2900$ & $0,777 \pm 0,013$ & $1 \mathrm{~h}$ à $210^{\circ} \mathrm{C}$ & & $0,28 \pm 0,03$ & \\
\hline \multirow[t]{4}{*}{ LT4 } & 1 & 68 & $505 \pm 60$ & 1636 & $123200 \pm 3000$ & $0,777 \pm 0,013$ & aucun & 0,94 & $0,18 \pm 0,02$ & $7,05 \pm 0,2$ \\
\hline & 2 & 72 & $440 \pm 50$ & 1542 & $116200 \pm 3000$ & $0,777 \pm 0,013$ & aucun & & $0,18 \pm 0,02$ & \\
\hline & 1 & 88 & $470 \pm 50$ & 1481 & $92800 \pm 2500$ & $0,777 \pm 0,013$ & $1 \mathrm{~h}$ à $210^{\circ} \mathrm{C}$ & 0,96 & $0,24 \pm 0,03$ & \\
\hline & 2 & 88 & $670 \pm 70$ & 2850 & $119000 \pm 2000$ & $0,777 \pm 0,013$ & $1 \mathrm{~h}$ à $210^{\circ} \mathrm{C}$ & & $0,26 \pm 0,03$ & \\
\hline \multirow[t]{3}{*}{ LT5 } & 1 & 411 & $3240 \pm 160$ & 5001 & $248900 \pm 3500$ & $1,369 \pm 0,013$ & aucun & 0,85 & $1,07 \pm 0,06$ & $7,7 \pm 0,2$ \\
\hline & 2 & 1480 & $5600 \pm 150$ & 3687 & $274000 \pm 4500$ & $1,369 \pm 0,030$ & aucun & 0,88 & $1,05 \pm 0,05$ & \\
\hline & 2 & 1017 & $6250 \pm 200$ & 2506 & $309600 \pm 6200$ & $1,369 \pm 0,030$ & $1 \mathrm{~h}$ à $210^{\circ} \mathrm{C}$ & 1,06 & $1,65 \pm 0,16$ & \\
\hline \multirow[t]{2}{*}{ LT6 } & 1 & 85 & $740 \pm 80$ & 3332 & $314200 \pm 5400$ & $3,213 \pm 0,023$ & $2 \mathrm{~h}$ à $215^{\circ} \mathrm{C}$ & & $0,45 \pm 0,05$ & $4,4 \pm 0,1$ \\
\hline & 2 & 94 & $720 \pm 70$ & 3493 & $329400 \pm 5600$ & $3,213 \pm 0,023$ & $2 \mathrm{~h}$ à $215^{\circ} \mathrm{C}$ & 1,01 & $0,42 \pm 0,04$ & \\
\hline \multirow[t]{4}{*}{ LT7 } & 1 & 46 & $275 \pm 40$ & 1621 & $122300 \pm 3000$ & $0,777 \pm 0,013$ & aucun & 0,66 & $0,10 \pm 0,02$ & $6,2 \pm 0,2$ \\
\hline & 2 & 40 & $240 \pm 40$ & 1824 & $105900 \pm 2500$ & $0,777 \pm 0,013$ & aucun & & $0,11 \pm 0,02$ & \\
\hline & 1 & 83 & $630 \pm 70$ & 1456 & $109500 \pm 2900$ & $0,777 \pm 0,013$ & $1 \mathrm{~h}$ à $215^{\circ} \mathrm{C}$ & 1,00 & $0,27 \pm 0,03$ & \\
\hline & 2 & 70 & $510 \pm 60$ & 1780 & $81100 \pm 1900$ & $0,777 \pm 0,013$ & $1 \mathrm{~h}$ à $215^{\circ} \mathrm{C}$ & & $0,29 \pm 0,03$ & \\
\hline \multirow[t]{5}{*}{ LT8 } & 1 & 35 & $270 \pm 50$ & 1388 & $104950 \pm 2800$ & $0,777 \pm 0,013$ & aucun & 0,73 & $0,12 \pm 0,02$ & $5,6 \pm 0,2$ \\
\hline & 2 & 52 & $280 \pm 40$ & 1854 & $116600 \pm 2700$ & $0,777 \pm 0,013$ & aucun & & $0,11 \pm 0,01$ & \\
\hline & 1 & & & & & & $1 \mathrm{~h}$ à $210^{\circ} \mathrm{C}$ & 0,94 & & \\
\hline & 1 & 89 & $650 \pm 70$ & 1609 & $100700 \pm 2500$ & $0,777 \pm 0,013$ & $2 \mathrm{~h}$ à $215^{\circ} \mathrm{C}$ & 1,03 & $0,30 \pm 0,03$ & \\
\hline & 2 & 104 & $700 \pm 70$ & 2036 & $114000 \pm 2500$ & $0,777 \pm 0,013$ & $2 \mathrm{~h}$ à $215^{\circ} \mathrm{C}$ & & $0,29 \pm 0,03$ & \\
\hline \multicolumn{11}{|l|}{ Inguapi } \\
\hline \multirow[t]{2}{*}{11} & 1 & 85 & $580 \pm 60$ & 2698 & $203400 \pm 3900$ & $1,062 \pm 0,013$ & aucun & 0,81 & $0,18 \pm 0,20$ & $4,4 \pm 0,1$ \\
\hline & 1 & 61 & $450 \pm 60$ & 1046 & $131400 \pm 4100$ & $1,062 \pm 0,013$ & $1 \mathrm{~h}$ à $210^{\circ} \mathrm{C}$ & 1,05 & $0,25 \pm 0,03$ & \\
\hline \multirow[t]{2}{*}{ I2 } & 1 & 334 & $5100 \pm 280$ & 2246 & $169500 \pm 3600$ & $1,062 \pm 0,013$ & aucun & 0,81 & $1,91 \pm 0,08$ & $7,4 \pm 0,2$ \\
\hline & 1 & 656 & $4960 \pm 200$ & 1679 & $122000 \pm 3000$ & $1,062 \pm 0,013$ & $1 \mathrm{~h}$ à $210^{\circ} \mathrm{C}$ & 1,06 & $2,58 \pm 0,12$ & \\
\hline \multirow[t]{3}{*}{ I3 } & 2 & 827 & $6580 \pm 230$ & 2816 & $380600 \pm 7200$ & $1,726 \pm 0,037$ & $1 \mathrm{~h}$ à $210^{\circ} \mathrm{C}$ & 0,88 & $1,78 \pm 0,06$ & $6,8 \pm 0,2$ \\
\hline & 1 & 690 & $5200 \pm 200$ & 1653 & $207700 \pm 5100$ & $1,726 \pm 0,037$ & $2 \mathrm{~h}$ à $215^{\circ} \mathrm{C}$ & 0,97 & $2,59 \pm 0,12$ & \\
\hline & 2 & 640 & $5120 \pm 200$ & 1658 & $203800 \pm 5000$ & $1,726 \pm 0,037$ & $2 \mathrm{~h}$ à $215^{\circ} \mathrm{C}$ & & $2,60 \pm 0,10$ & \\
\hline
\end{tabular}

Nf et Ni sont les nombres de traces de fission spontanée et induite comptées ; $\rho$ s et $\rho$ i les densités de traces, en nombre de traces par $\mathrm{cm}^{2}$; $\Phi$ la fluence neutronique, en neutrons $/ \mathrm{cm}^{2}$; Ds/Di, le rapport des diamètres moyens de traces de fission spontanée et induite ; $\mathrm{t}$ l'âge TF, en millions d'années ; Cu, la teneur en Uranium. Dans l'équation (1), $A=\omega \mathrm{l} / \lambda$ où $\sigma=580 \times 10^{-24} \mathrm{~cm}^{2}$ est la section efficace de capture des neutrons thermiques par $20 \mathrm{U}, \mathrm{I}=7,252 \times 10^{-3}$ le rapport des abondances isotopiques ${ }^{25} \mathrm{U} /{ }^{200} \mathrm{U}$ et $\mathrm{I}=7,03 \times 10^{-17}$ ans $s^{-1}$ la constante de décroissance par fission spontanée de $2{ }^{20} \mathrm{U}$. La justification de cette valeur est donnée dans Bigazzi et al. (1988 a). $\Phi$ a été dérivé à partir de verres étalons du NIST (voir texte), comme dans Poupeau et al. (1992, 1993).

L'incertitude sur les densités de traces mesurées a été calculée selon une loi de Poisson, avec $\sigma \rho / \rho=1 /(\mathrm{N})^{1 / 2}$, où $\mathrm{N}$ est le nombre de traces comptées; l'incertitude sur

les âges, selon Bigazzi et al. (1986b). de verre étalon (NIST) de teneur en Uranium connue et péch la densité de traces dans un détecteur externe de traces associé à un échantillon d'obsidienne, ces deux montages ayant été irradiés au préalable conjointement en réacteur nucléaire.

miné par deux observateurs, on constate la cohérence des résultats. Ainsi, pour l'obsidienne LT5, les valeurs de $D_{f} / D_{j}$ obtenues par chacun des observateurs sont-elles respectivement de $0,85 \pm 0,03$ et $0,88 \pm 0,03$ pour l'échantillon non traité thermiquement.
L'âge vrai des obsidiennes a été obtenu par la technique des âgesplateaux. Après un recuit de 1 à 2 heures à une température de $210{ }^{\circ} \mathrm{C}$ ou $215^{\circ} \mathrm{C}$, les rapports $D_{f} / D_{i}$ ont été ramenés à l'unité. Seuls deux échantillons, LT8 et I3, ont dû subir deux recuits pour y parvenir. Cing échantillons de La Tolita qui présentent des âges apparents s'échelonnant de $0,10 \mathrm{Ma}$ à $0,19 \mathrm{Ma}$ et des degrés d'effacement variables des traces fossiles ont alors atteint des âges-plateaux concordants à 0,28 $0,30 \mathrm{Ma}$. Quatre d'entre eux, LT2, LT3, LT7 et LT8, qui ont des teneurs 
semblables en Uranium, comprises entre 5,5 et $6,2 \mathrm{ppm}$, pourraient appartenir à la même coulée ; le cinquième, avec 3,9 ppm d'Uranium, se distingue de ce groupe. Un sixième échantillon, d'âge apparent $0,18 \mathrm{Ma}$, est caractérisé par un âge-plateau marginalement concordant (à $2 \sigma$ près) avec les précédents, à environ $0,25 \mathrm{Ma}$, mais il possède une teneur en Uranium significativement plus élevée, de 7,05 ppm.

Seules les obsidiennes LT5 et LT6 se distinguent nettement du groupe d'âges-plateaux précédents, avec respectivement $1,65 \pm 0,07 \mathrm{Ma}$ et $0,42 \pm 0,04 \mathrm{Ma}$ et représentent donc deux autres sources distinctes. II y aurait ainsi, à La Tolita des obsidiennes provenant d'au moins trois sources, caractérisées par des âges de respectivement $0,25-0,30 \mathrm{Ma}$, $0,42 \mathrm{Ma}$ et 1,65 Ma.

Les trois échantillons d'Inguapi se répartissent en deux groupes d'âges. L'artefact 11 semble appartenir au groupe principal de La Tolita, avec un âge apparent de 0,18 Ma, un âgeplateau de 0,25 $\mathrm{Ma}$ et une teneur en Uranium de 4,4 ppm. Les échantillons 12 et 13 sont par contre deux représentants d'un quatrième groupe d'âges-plateaux, vers 2,58-2,60 Ma. Leurs teneurs en Uranium, relativement proches, de respectivement 7,4 et $6,8 \mathrm{ppm}$, n'excluent pas que ces deux dernières pièces puissent parvenir d'une même coulée.

\section{DISCUSSION}

Les onze artefacts que nous avons étudiés se présentaient sous la forme de pièces d'obsidienne de couleur foncée. Leur couleur, leur type de cassure, conchoïdal, et l'absence d'inclusions minérales, ne permettaient pas de les distinguer nettement les uns des autres. II apparaît cependant, à la lumière des datations obtenues, que ces obsidiennes proviennent d'au moins quatre sources clairement différenciées, caractérisées par des âges d'émission d'obsidienne situés vers $0,25-0,30 \mathrm{Ma}$, $0,42 \mathrm{Ma}, 1,65 \mathrm{Ma}$ et 2,59 Ma.

\section{- Comparaison avec les données régionales}

II n'existe actuellement de données TF que sur un seul autre site côtier de la région. II s'agit de celui de Palmar, situé à environ $350 \mathrm{~km}$ au sud de La Tolita. Les fouilles conduites par Bischof (1975 a, b) à Palmar ont révélé un site occupé de 1200 BC, au Formatif final, jusqu'au stade des Développements régionaux, vers 300-500 AD. Miller et Wagner (1981) ont étudié 24 artefacts en obsidienne de ce site. Ces auteurs ont remarqué que les densités de traces de fission fossiles permettaient de répartir ces artefacts en deux groupes, et ont daté deux représentants de chacun d'eux. Deux de ces artefacts présentent des caractéristiques TF qui permettent de les rattacher à notre groupe majeur de La Tolita : âges apparents, âgesplateaux et teneurs en Uranium de respectivement $0,14 \mathrm{Ma}, 0,25 \mathrm{Ma}$, $5,6 \mathrm{ppm}$ pour l'artefact 094E, et $0,15 \mathrm{Ma}, 0,25 \mathrm{Ma}$ et 5,9 ppm pour le 094M. Les deux autres échantillons datés définissent un nouveau groupe, avec des âges apparents de respectivement 1,31 $\mathrm{Ma}$ et 1,25 $\mathrm{Ma}$, des âges-plateaux de 2,01 $\mathrm{Ma}$ et $1,89 \mathrm{Ma}$, et des teneurs en Uranium de 6,9 et $6,6 \mathrm{ppm}$.

Les sources potentielles d'obsidienne les plus proches se trouvent dans la zone de la Sierra. Le volcanisme acide néogène s'y est développé à partir de l'Oligocène et surtout du Miocène, jusqu'à l'actuel. En Colombie et en Équateur, ce volcanisme est concentré dans la Dépression Centrale (fig. 4). Plus au Sud, au Pérou, en Bolivie et en Argentine, on le rencontre dans l'Altiplano. Enfin au Chili il est limité à la Cordillère Occidentale jusque vers le trente-cinquième degré de latitude Sud.

On ne dispose actuellement que de données très limitées sur l'âge des obsidiennes de la chaîne andine. En Colombie, aucune coulée ne semble encore avoir été datée par traces de fission ; le seul artefact en obsidienne daté par TF, celui du site archéologique de Cerro la Tefa près de Cali, présente un âge-plateau de $2 \mathrm{Ma}$; par ailleurs, cet artefact semble être passé au feu il y a environ 3600 ans (Miller et Wagner, 1981), c'est-à-dire vers le début du Formatif ou juste avant.

En Équateur, le principal centre d'émission d'obsidienne se situe à proximité de Quito (Salazar, 1985). Les premières datations d'obsidiennes "géologiques" de cette région sont celles de Miller et Wagner (1981), avec des âges-plateaux de respectivement $1,81 \mathrm{Ma}$ et $1,78 \mathrm{Ma}$ pour deux échantillons de provenance non précisée. Ces mêmes auteurs avaient aussi obtenu comme âges apparents de deux obsidiennes de Mullumica 0,19 $\mathrm{Ma}$ et 0,21 Ma, c'est-à-dire des valeurs équivalentes à celles mesurées par Bigazzi et al. (1992) dans leur groupe d'âges le plus récent (voir ci-dessous). Miller et Wagner ont aussi daté trois artefacts en obsidienne du même secteur: deux d'entre eux, qui proviennent du site archéologique d'El Inga, à $17 \mathrm{~km}$ de la coulée de Mullumica, présentent des âges de respectivement 0,00206 Ma (âge corrigé par la méthode des diamètres de traces de Storzer, 1970) et 1,72 Ma (âge-plateau) ; un troisième, du site de La Esperanza (au Nord d'El Inga), est daté à $0,21 \mathrm{Ma}$.

Les seules informations directes sur l'âge des gisements naturels des obsidiennes équatoriennes sont celles apportées par Bigazzi et al. (1992) pour l'Est et le Sud-Est immédiats de Quito (fig. 4), avec la datation par TF de douze échantillons, certains provenant de sites exploités au cours des périodes Formative et des Développements Régionaux, comme à Quiscatola et Mullumica (Salazar, 1985, 1992). Les résultats montrent un groupe d'âges-plateaux vers 0,2 Ma, avec quatre échantillons des coulées de Mullumica, deux de celle de Callejones et un du volcan de Potrerillos ; un second groupe, entre 1,4 Ma-1,7 Ma est défini par les âges du dépôt secondaire ("ancient debris flow") de Rodeo Corrales et les témoins très érodés de brèches à obsidienne de Cerro Yanaurcu et Loma Quiscatola. Les deux derniers échantillons mesurés dans ce travail présentent des âges intermédiaires, vers $0,4 \mathrm{Ma}$ pour le pointement blocs d'obsidienne de Yurac Pacha et 0,85 Ma pour la coulée d'El Tablon. Enfin, à proximité d'El Inga (à $5 \mathrm{~km}$ au NW de Pintag), deux pièces d'obsidienne associées à une coulée pyroclastique, datées par Arias et al. (1986) et considérées par ces auteurs comme des éclats naturels, puis réinterprétées par Bigazzi et al. (1992) comme des artefacts archéologiques provenant d'un atelier de taille (après réinspection du site par M. Cortelli ; G. Bigazzi, comm. pers.), possèdent des âges TF corrigés par la technique des diamètres de traces et/ou du plateau, de respectivement $0,32 \pm 0,02 \mathrm{Ma}$ (moyenne pondérée à $\pm 1 \sigma$ sur quatre déterminations) avec une teneur en Uranium de 
9,6 ppm, et 2,14 $\pm 0,08 \mathrm{Ma}$ (m.p. sur trois mesures) avec $9,7 \mathrm{ppm} \cup$ (teneurs en $U$ déterminées par TF ; G. Bigazzi, communication personnelle, 1993).

Les seules autres datations TF (corrigées par la méthode des diamètres ou celle des âges-plateaux) d'obsidiennes andines sont, d'une part, dans le Sud du Pérou, celles des verres ignimbritiques du "sillar" d'Arequipa, de 2,40 $\pm 0,02 \mathrm{Ma}$ (Vatin-Pérignon et al., 1994) et des macusanites de la région de Puno, d'âges compris entre 4,7 $\mathrm{Ma}$ et 7,2 Ma (Poupeau et al., 1992, 1993 et références incluses), et d'autre part, en Bolivie, celle d'une obsidienne de provenance inconnue, vers 5,1 Ma (Miller et Wagner, 1981).

\section{- Les obsidiennes de La Tolita et Inguapi : une origine andine?}

Le survol des données géochronologiques par TF sur les obsidiennes de la Sierra de Colombie et d'Équateur fait apparaître le caractère très fragmentaire des connaissances actuelles, puisqu'on ne dispose que d'un âge en Colombie et de moins d'une vingtaine pour l'Équateur, tous relatifs aux gisements de la région de Quito. Toutefois, il ressort de cette revue une mise en évidence d'épanchements d'obsidiennes depuis au moins $2 \mathrm{Ma}$ et jusqu'à l'actuel. L'échantillonnage analysé fait de plus ressortir l'existence de deux groupements d'âges, vers respectivement $0,17-0,43 \mathrm{Ma}$ et 1,39-2,14 Ma (fig. 5). Le premier de ces groupes comporte huit échantillons géologiques et deux artefacts provenant de la région cerclée dans la carte de la figure 4, ainsi qu'un artefact du site de La Esperanza, au Nord de cette région ; le second, cinq obsidiennes et deux artefacts de la région de Quito, ainsi qu'un artefact des environs de Cali en Colombie.

Ces deux groupes renferment aussi (zone grisée de la figure 5), les âges de treize artefacts sur quinze des sites côtiers de La Tolita, Inguapi et Palmar. Cette similitude entre la distribution des âges des obsidiennes de la Sierra et celle des régions côtières suggère que l'hypothèse d'une origine locale de la matière première des artefacts des

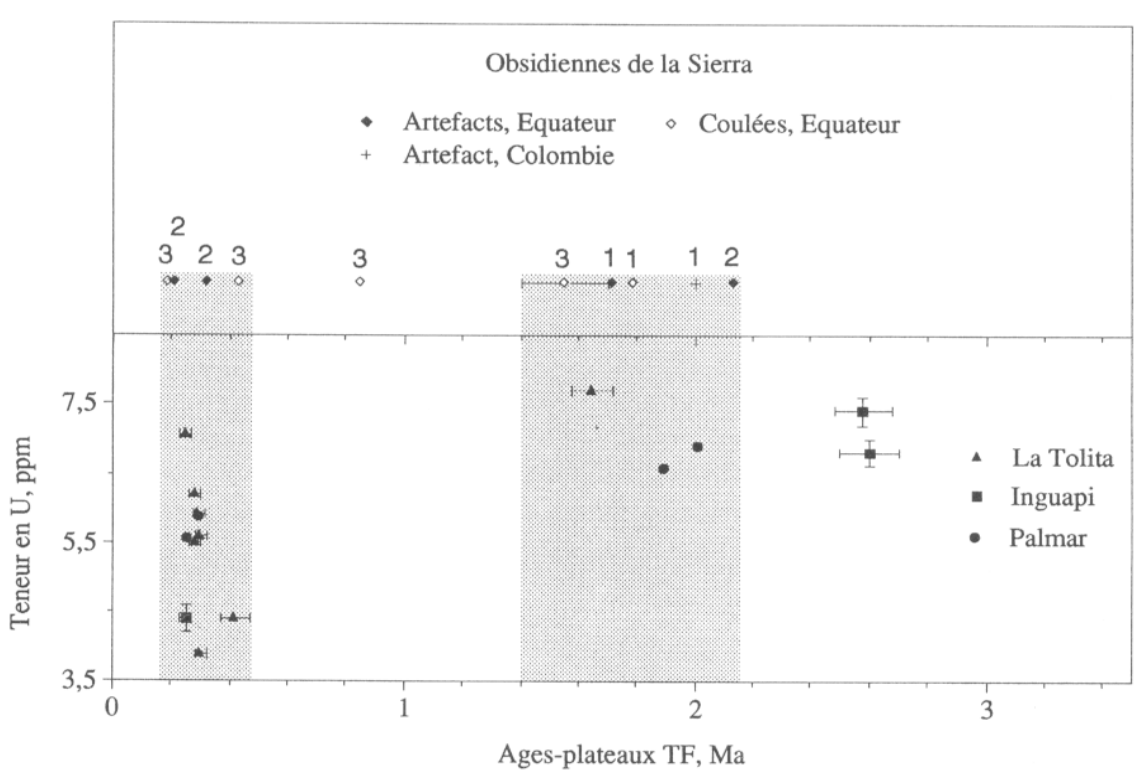

Fig. 5 - Ages TF corrigés (méthode des diamètres ou des âges-plateaux) des obsidiennes de Colombie et d'Equateur.

Haut : obsidiennes de la Sierra, prélevées soit dans sites archéologiques, soit dans des gisements naturels, primaires ou secondaires. Les chiffres au-dessus des symboles se référent aux articles d'où ont été extraites les données : 1, Miller et Wagner (1981) ; 2, Arias et al. (1986) ; 3, Bigazzi et al. (1992).

Bas : Échantillons des sites côtiers équatoriens de Palmar (Miller et Wagner, 1981), de La Tolita et du site colombien d'Inguapi (ce travail).

sites de La Tolita, Inguapi et Palmar mérite d'être considérée.

Le groupe des âges les plus récents de la figure 5 est le mieux représenté dans les sites examinés ici, avec sept artefacts pour La Tolita, deux pour Palmar et un pour Inguapi. Les âges de ce groupe mesurés dans les sites côtiers sont représentés dans la Sierra : le groupe d'âges 0,24-0,31 Ma, d'une part, est proche de celui déterminé pour l'artefact prélevé près de Pintag (fig. 4) et daté par Arias et al. (1986) à 0,32 $\pm 0,02 \mathrm{Ma}$; d'autre part, l'âge de $0,43 \pm 0,04 \mathrm{Ma}$ de l'échantillon LT6 est concordant avec celui de l'obsidienne de Yurac Pacha datée par Bigazzi et al. (1992) à 0,42 $\pm 0,05 \mathrm{Ma}$. Les données géochronologiques, avec une précision $( \pm 2 \sigma)$ de $\pm 0,06 \mathrm{Ma}$ sur des âgesplateaux de 0,25 - 0,30 Ma, ne permettent cependant pas de conclure ou non à une unicité de source de matière première. La grande variation des teneurs en Uranium dans ce groupe d'âges, comprises entre $3,9 \pm 0,2 \mathrm{ppm}$ et $7,05 \pm 0,2 \mathrm{ppm}$ suggère de son côté une diversité de sources. Six échantillons (4 pour La Tolita, 2 pour Palmar), avec des teneurs très proches, entre 5,5 et $6,2 \mathrm{ppm}$, pourraient cependant provenir d'une même coulée.

Dans le second groupe d'âges en grisé de la figure 5 , celui de $1,65 \pm 0,16 \mathrm{Ma}$ de l'échantillon de La Tolita LT5 est compatible avec ceux de plusieurs obsidiennes de la Sierra, notamment celle de Cerro $\mathrm{Ya}$ naurcu, d'environ 1,5 Ma. A ce groupe appartiennent aussi les deux artefacts de Palmar datés par Miller et Wagner (1981).

Le seul groupe d'âges d'un site côtier, celui de La Tolita, non représenté dans l'échantillonnage andin analysé jusqu'à présent reste celui de 2,6 Ma.

La présence d'obsidiennes situées dans la chaîne andine, à moins de $150 \mathrm{~km}$ de la côte, et présentant des âges TF concordants avec ceux des artefacts de sites côtiers, suggère qu'une recherche complémentaire utilisant des méthodes géochimiques permettrait d'établir définitivement l'origine des obsidiennes des sites côtiers de la région Nord-Équateur/Sud-Colombie. En effet, on admet en général que toute obsidienne présente une composition chimique unique (Cann, 1983 ; Goffer, 1983). En application de ce principe, un groupe de Berkeley avait dès 1981 analysé, par activation neutronique instrumentale et fluorescence de rayons- $X, 40$ obsidiennes équatoriennes. Leurs échantillons provenaient de quatre sites archéologiques de la région de Quito, ainsi que de La Tolita et des coulées de Mullumica. La Tolita n'était représentée que par un seul échantillon : un bloc d'obsidienne sur lequel avaient été prélevés 5 fragments pour ana- 
lyses. Ces 40 échantillons définissent 6 groupes géochimiques. Celui auquel appartient l'obsidienne de La Tolita, le groupe "Ecuador B", est constitué d'échantillons de trois sites archéologiques, dont celui de Cotocollao, et d'un autre site, "Quito", qui appartient à la période de l'Intégration. Les obsidiennes de ces sites présentent une composition différente de celle des obsidiennes des coulées de Mullumica (Asaro et al., 1981 a).

Un second travail, portant sur 24 échantillons supplémentaires, permit à Asaro et al. (1981 b) de montrer que le groupe géochimique Ecuador B était aussi présent par des artefacts d'obsidienne dans les sites de Nayon Sur (Période formative, dans Quito) et de Santa Marta (site précéramique, vallée de Quito). Ces nouvelles analyses permettaient d'exclure comme source géologique des artefacts du groupe Ecuador B non seulement l'obsidienne de Mullumica, mais encore celles de Yanaurco et Quiscatola.

L'analyse géochimique, comme les datations TF, suggère donc fortement que les obsidiennes de La Tolita pourraient, au moins pour partie, avoir leur source dans l'intérieur du continent, voire dans le complexe volcanique de la région située au SE de Quito. Dans I'hypothèse où les obsidiennes des sites côtiers de la Période Formative et de celle des Développements Régionaux seraient venues de la Sierra, comment ce matériau aurait-il diffusé et sous quelle(s) forme(s) ? Certains artefacts auraient pu être façonnés directement sur le site de prélèvement : ainsi plusieurs ateliers de taille sont connus dans la région de Mullumica (Salazar, 1985, 1992) ; d'autres auraient pu circuler sous forme de nodules dégrossis, comme le bloc de La Tolita analysé par Asaro et al. (1981 a). S'agissait-il d'expéditions destinées à la collecte de la "roche noire", ou bien faut-il penser la circulation de l'obsidienne, dans un réseau avec ses relais organisés, conjointement à d'autres échanges ?

Des réponses à certaines de ces questions pourraient se dégager si, en parallèle de la poursuite des datations par TF de pièces archéologiques, pouvait être repris un travail de prospection et de datation d'obsidiennes géologiques de Colombie et d'Équateur. En outre il serait particulièrement intéressant d'associer à la géochronologie par TF d'autres méthodes de caractérisation géochimique de ces verres volcaniques afin de disposer de définitions plus fines de chaque source potentielle de matière première pour l'industrie lithique.

\section{CONCLUSION}

La datation par traces de fission de onze artefacts d'obsidienne des sites côtiers de La Tolita et d'Inguapi montre que plusieurs sources de matière première sont impliquées. D'après la distribution des âges, au moins trois sources doivent être invoquées pour La Tolita, deux pour Inguapi. Une source d'obsidienne, caractérisée par un âge d'environ 0,25-0,30 Ma et une teneur en Uranium entre 5,5 et 6,2 ppm d'Uranium, pourrait être représentée à la fois par des artefacts de La Tolita et de Palmar, autre site côtier à environ $350 \mathrm{~km}$ plus au Sud.

Par ailleurs, une comparaison avec les âges TF disponibles pour les obsidiennes de la Sierra montre que treize des quinze âges TF des artefacts d'obsidienne des trois sites côtiers considérés coïncident avec deux épisodes de volcanisme marqués par des épanchements d'obsidienne considérés comme les plus importants de l'Équateur (Salazar, 1985), au SE de Quito. II apparaît donc comme possible que les artefacts d'obsidienne des sites côtiers de la Période Formative, comme de celle des Développements Régionaux, puissent avoir une origine locale, et qu'il ne soit plus justifié d'invoquer une ou des sources méso-américaines.

Les âges TF de deux artefacts de La Tolita, avec 2,6 Ma, n'ont, pour le moment, aucune contrepartie dans la Sierra. Cela n'implique pas nécessairement une origine plus lointaine, et d'autres hypothèses sont plausibles, comme l'épuisement d'un gisement par exploitation anthropique (Bigazzi et al. 1990), sa dissimulation par la végétation, ou en raison d'enfouissement sous des dépôts volcaniques ou sédimentaires plus récents. Enfin, il ne faut pas négliger l'érosion glaciaire, qui a déjà fortement réduit certaines coulées (Bigazzi et al., 1992).

Asaro et coll. (1981 a, b ; Burger et Asaro, données non publiées,
1977) avaient décelé, dans un travail pionnier, six groupes géochimiques discrets dans les obsidiennes de la région de Quito. L'appartenance d'un bloc d'obsidienne récolté à La Tolita à l'un de ces groupes, défini par la composition chimique de ce bloc et de quelques artefacts provenant de quatre sites proches de Quito, suggérait également une possible origine andine pour cette obsidienne.

Datation par TF et caractérisation géochimique s'accordent donc à suggérer une origine locale (dans la Sierra) à la matière première des artefacts en obsidienne des sites côtiers des périodes Formative et des Développements Régionaux. L'une des tâches à accomplir, si l'on veut mieux comprendre la systématique de l'exploitation et de la circulation de l'obsidienne dans cette région des Andes à l'époque considérée, consistera sans nul doute à associer étroitement, dans le futur, approche géochronologique et approche géochimique à l'étude proprement archéologique des sites et de leurs industries lithiques.

\section{Remerciements}

Les auteurs expriment leur gratitude envers G. Bigazzi, qui leur a aimablement communiqué ses résultats avant publication, G. Bigazzi et E. Salazar pour leur avoir transmis des informations complémentaires sur les sites archéologiques et les gisements d'obsidienne équatoriens, $\mathrm{M}$. Bonhomme et $\mathrm{N}$. Vatin-Pérignon pour leurs informations sur le volcanisme néogène andin. Ce travail a été en partie financé par le GDR/CNRS n¹033 "Méthodes Nucléaires en Archéologie".

\section{Bibliographie}

Alcina-Franch J., Alonso A., Bouchard J.F. et GUINÉA M. (1987) - Navegación precolombina ; el caso del litoral pacifíco ecuatorial : evidencias e hipótesis. Revista Española de Antropologia americana, $\mathrm{n}^{\circ}$ XVII, Madrid, p. 35-73.

Arias C., Bernardes C., Bigazzi G., Bonadonna F.P., César M.F., Hadler-Neto J.C., LatTes C.M.G., Oliveira J.X., Osorio-ARAYA A.M. et RADI G. (1986) - Identificaçao de obsidianas atraves da dataçao com o método dos traços de fissao. Ciência e Cultura, 38, p. 285-308.

Asaro F., Michel H.V. et Burger R.L. (1981 a) - Chemical source groups in ecuadorian obsidian. Lawrence Berkeley Laboratory Report LBL-13247, $27 \mathrm{p}$. 
Asaro F., Michel H.V. et Burger R.L. (1981 b) - Major sources of ecuadorian archaeological obsidian and provenience assignment of artifacts. Lawrence Berkeley Laboratory Report $L B L-13246,18 \mathrm{p}$.

BeLl R.E. (1965) - Investigaciones Arquelógićas en el sitio de El Inga, Ecuador. Édition bilingue, anglaisespagnol ; Casa de la Cultura Ecuatoriana, Quito.

Bigazzi G., Meloni S., Oddone M. et RADI G. (1986 a) - Provenance studies of artefacts : trace elements analysis and data reduction. J. Radioanal. Nucl. Chem., 98, p. 353-363.

Bigazzi G., Bonadonna F. et Hader Neto J.C. (1986 b) - Contribution to statistics for fission track counting. $\mathrm{NuCl}$. Tracks, 3, p. 123-126.

Bigazzı G., Hadler Neto J.C., Norelli P., Osorio Araya A.M., Paulino R., PouPEAu G. et Stella de Naval L. (1988) - Dating of glass : the importance of correctly identifying fission tracks. Nucl. Tracks Radiat. Meas., 15, p. 711714.

Bigazzi G., Marton P., Norelli P. et RozLOZNIK (1990) - Fission track dating of carpathian obsidians and provenance identification. Nucl. Tracks Radiat. Meas., 17, p. 391-396.

BIgazZı G., HADLER J.C., IUNES P.J. et OSORIO A.M. (1991) - Fission track Ds/Di measurements in artificial glass under conditions free from fading and radiation. Nucl. Instr. Methods Phys. Res., B53, p. $67-70$

Bigazzı G., Coltellı M., Hadler N.J.C., Osorio araya A.M., Oddone M., SalaZAR E. (1992) - Obsidian-bearing lava flows and pre-Columbian artifacts from the Ecuadorian Andes : First new multidisciplinary data. J. South Amer. Earth Sci., 6, p. 21-32.

Bischof H. (1975 a) - La fase Engoroy. Periodos, Cronologia y Relaciones. In Estudios sobre la arqueología del Ecuador, BAS 3, Éd. U. Oberem, Bonn, p. 15-38.

BIsCHOF H. (1975 b) - El Machalilla temprano y algunos sitios cercanos a Valdivia. In Estudios sobre la arqueología del Ecuador, BAS 3, Éd. U. Oberem, Bonn, p. 43-63.

BOUCHARD J.-F. (1984) - Recherches archéologiques dans la région de Tumaco (Colombie). Mémoire $n^{\circ} 34$; Institut Français d'Études Andines, Éditions Recherche sur les civilisations, A.D.P.F., Paris, 205 p.

Bouchard J.-F. (1986) - Las más antiguas culturas precolombinas del pacifico ecuatorial septentrional. Simposio del 45 Congreso Internacional de Americanistas : Arqueologia y Etnohistoria del Sur de Colombia y Norte del Ecuador. Miscelanea Antropológica Ecuatoriana, Quito, n 6, p. 109-129.

BOUCHARD J.-F. (1989 a) - Archéologie Préhispanique du littoral Pacifique Nord-Équatorial. Bull. Soc. Préhist. Fr. vol. 10/12, p. 393-396.
BOUCHARD J.-F. (1989 b) - Evidencias de relaciones interculturales en la región norte (sur de Colombia y Norte del Ecuador). Relaciones interculturales en el área ecuatorial del Pacifico durante la época precolombina. Proceedings 46 Congreso Internacional de Americanistas, Amsterdam, 1988 B.A.R. International Series $n^{\circ} 503$, Oxford, p. 29-44.

CANN J.R. (1983) - Petrology of obsidian artefacts. In The Petrology of Archaeological Artefacts, Eds. D.R.C. Kempe et A.P. Harvey, Clarendon Press, Oxford, p. 227-255.

Cubillos J.C. (1955) - Tumaco : notas arqueológicas. Ministerio de Educación, Bogota, $145 \mathrm{p}$.

DÉBoer W. et Tolstoy P. (1989) - An arquaeological sequence for the Santiago-Cayapas River basin, Esmeraldas, Ecuador. J. Field Archaeology, 16, p. 295-308.

FERdON F. et CORBETT J. (1941) - Depósitos arqueológicos de La Tolita. Bol. Acad. Nac. Historia, Quito, 21, p. 5-15.

Fleischer R.L., Price E P.B., Walker R.M et LEAKEY L.S.B. (1965 a) - Fission track dating of a mesolithic knife. $\mathrm{Na}$ ture, 205, 1138.

Fleischer R.L., Price P.B., Walker R.M. et LEAKEY L.S.B. (1965 b) - Fission track dating of bed I, Olduvai Gorge. Science, 148 , p. $72-74$

Fleischer R.L., Price P.B., et Walker R.M. (1975) - Nuclear tracks in Solids: Principles and Applications. University of California Press, Berkeley, 604 p.

Gleadow A.J.W. (1980) - Fission track age of the KBS tuff and associated hominid remains in northern Kenya. Nature, 284, p. 225-230.

Gleadow A.J.W. (1981) - Fission track dating methods : what are the real alternatives ? Nucl. Tracks, 5, p. 3-14.

GoFfER Z. (1983) - Physical studies of archaeological materials. Rep. Progr. Phys., 46, p. 1193-1234.

Guo Shilun, Zhou Shuhua, Meng Wu, Zhang Pengfa, HaO XiuHong, LIU ShunSHENG, ZHANG FENG, Hu RUIYING et LIU JINGFA (1980) - Fission track dating of Peking man. Kexue Tongba, 25, p. 770-772.

Guo Shilun, Liu Shungsheng, Sun Shengfen, Zhang Fen, Zhou Shuhua, HaO XUIHONG, Hu RUIYING, MENG WU, ZHANG PENGFA et LiU JINFA (1990) - Age and duration of Peking man site by fission track method. 15th Intern. Conf. on Particle Tracks in Solids, Marbourg, résumé.

HURFord A.J. et WATKINS R.T. (1987) Fission-track age of the tuffs of the Buluk member, Bakate formation, Northern Kenya : a suitable fission-track age standard. Chem. Geol. (Isot. Geosci. Sect.), 66, p. 209-216.

MAYER-OAKES W.J. (1986) - EI Inga, a Paleo-Indian site in the Northern Sierra of Ecuador. Trans. Amer. Phil. Soc., 76.
Meggers B. (1966) - Ecuador. Ancient Places and Series. Thames and Hudson, Londres, $220 \mathrm{p}$.

Miller D.S. et Wagner G.A. (1981) - Fission-track ages applied to obsidian artefacts from South America using the plateau-annealing and the track-size age-corrections techniques. Nucl. Tracks, 5, p. 147-155.

Poupeau G. (1979) - Datations par traces de fission de l'Uranium : principes et applications aux problèmes du Quaternaire. Bull. Ass. Fr. Quaternaire, $n^{\circ}$ 58-59, p. 15-26.

Poupeau G. (1982) - Precision, meaning and accuracy of fission track dating. In "Nuclear Tracks", Indian Acad. Sci., Bangalore, p. 67-100.

poupeau G., Sabil N., Villa I.M., BigazzI G., Vatin-Pérignon N., Flores P., PÉReYra P., Salas G. et ARROYo G. (1992) - Fission-track and $\mathrm{K}$-Ar ages of "macusanite" obsidian glasses, (SE Peru) : geodynamic implications. Tectonophysics, 205, p. 295-305.

Poupeau G., Labrin E., Sabil N., Bigazzı G., Arroyo G. et VAtin-Pérignon (1993) - Fission track dating of 15 Macusanite glass pebbles from the Macusani volcanic field (SE Peru). Nuclear Tracks and Radiat. Meas., sous presse.

Poupeau G., Labrin E., Chaouche A., AKKOUCHE M. et KadDaRI B.M. (1994) Utilisation du réacteur nucléaire du Ministère Délégué aux Universités et à la Recherche Scientifique (Draria, Algérie), pour la datation par traces de fission. Rapport interne, $25 \mathrm{p}$.

Price P.B. and WALker R.M. (1963) Fossil tracks of charged particles in micas and the age of minerals. J. Geophys. Res. , 68, p. 4847-4862.

Reichel-Dolmatoff G. (1965) Colombia. Ancient People and Place Series. Thames and Hudson, Londres, $231 \mathrm{p}$.

Reichel-Dolmatoff G. (1987) - Arqueológia de Colombia : un texto introductorio. Fundación Segunda Expedición Botánica, Bogotá, 280 p.

SALAZAR E. (1985) - Investigaciones arqueológicas en Mullumica (Provincia del Pichincha). In Miscelanea Antropológica Ecuatoriana, Boletín de los Museos del Banco Central del Ecuador, Ano 5, n 5, p. 129-160.

SALAZAR E. (1992) - El intercambio de obsidiana en el Ecuador Precolombino : Perspectivas teorico-metodológicas. In Arqueológia en Americana Latina Hoy, Ed. G. Politis, Biblioteca del Banco Popular, Fondo de promoción de la Cultura, Bogóta, Colombie, p. 116-131.

STORZER D. (1970) - Fission track dating of volcanic glasses and the thermal history of rocks. Earth Planet. Sci. Letters, 8, p. 55-60.

Storzer D. et Poupeau G. (1973) - Agesplateaux de verres et de minéraux par 
la méthode des traces de fission. C. $R$ Acad. Sci. Paris, 276D, p. 137-139.

SUZUKI M. (1973) - Chronology of prehistoric human activity in Kanto, Japan. Part I: framework for reconstructing prehistoric human activity in obsidian. J. Fac. Sci. Tokyo University, IV, p. 41318

UHLE M. (1927 a) - Las antiguas civilizaciones esmeraldeñas. Anales de la Universidad Central, Quito, 38.

UHLE M. (1927 b) - Estudios esmeraldeños. Anales de la Universidad Central, Quito, 39, p. 1-61 et 107-136.

VALDEZ F. (1986) - Investigaciones arqueológicas en La Tolita (Esmeraldas). Simposio del 45 Congreso Internacional de Americanistas : Arqueologia y Etnohistoria del sur de Colombia y Norte del Ecuador. Miscelanea Antropológica Ecuatoriana, Quito, p. 81107.

VALDEZ F. (1987) - Proyecto Arqueológico La Tolita. Banco Central del Ecuador, Quito, $91 \mathrm{p}$.

Vatin-Pérignon N., Poupeau G., Oliver R., Labrin E., Keller F. et Bellot-Gurlet L. (1994) - Geochemical characterization of ignimbritic tuffs from the Western peruvian Cordillera and the bolivian Altiplano and fission track age of the sillar of Arequipa (southern Peru). J. South Amer. Earth Sci., soumis.

WAGNER G.A. (1978) - Archaeological applications of fission track dating. Nucl. Track Detection, 2, p. 51-63.

Watanabe N. et SuzUKI M. (1969) - Fission track dating of archaeological glass material from Japan. Nature, 222, p. 1057-1058.

\section{Bibliographie archéologique complémentaire}

ALCINA-FrAnCH J. (1979) - La arqueologia de Esmeraldas (Ecuador). Introducción general. Memorias de la Misión Arqueológica española en el Ecuador, vol. 1, Ministerio de Asuntos Exteriores, Madrid, $161 \mathrm{p}$

BOUCHARD J.-F. (1990) - Deux sites côtiers : Tumaco et La Tolita (ColombieÉquateur). Dossiers de l'archéologie, $n^{\circ} 145$, pp. 64-69.

GUINÉA M. (1986) - El Formativo de la región Sur de Esmeraldas visto desde el yacimiento de Chévele. Simposio del 45 Congreso Internacional de Americanistas : Arqueologia y Etnohistoria del Sur de Colombia y Norte del Ecuador. Miscelanea Antropológica Ecuatoriana, Quito, $n^{\circ} 6$, p. 19-46.

Lathrap D.W., Collier D. et Chandra $H$. (1975) - Ancient Ecuador : Culture, Clay and Creativity 3000 B-300 BC. Field Museum of Natural History, Chicago, $110 \mathrm{p}$.

Marcos J. (1984) - Breve Prehistoria de Ecuador. In Tesoros del Ecuador Antiguo. Instituto de Cooperación Iberoamerica, Madrid, p. 9-23.

Patino Castaño D. (1987) - Arqueología de la costa pacifica caucana. Bol. de Arqueología, Ano 2, Fundación de Investigaciones Arqueológicas Nacionales, Bogotá, p. 65-81.

Patino Castaño D. (1988) - Asentamientos prehispanicos en la costa pacífica caucana. Fundación de Investigaciones Arqueológicas Nacionales, Banco de la Republica, Bogotá, 160 p.

ReICHEL-Dolmatoff G. (1979) - Colombia indigena : período prehispánico. Manual de Historia de Colombia, vol. 1 Colcultura, Bogota, p. 31-114.

RIVÉRA M. (1979) - Ensayo de tipología de la cerámica Tiaone. Actes du $42^{\mathrm{e}}$ Congrès International des Américanistes, vol. 9A, Paris, p. 229-244.

Rivéra M., Sanchez Montañes E., Ciudad A., Rodriguez A. et Colon A. (1984) La cultura Tiaone. Memorias de la Misión Arqueológica Española en Esmeraldas, vol. 4, Ministerio de Asuntos Exteriores, Madrid, $383 \mathrm{p}$.

SAVILLE M. (1910) - Archaeological researches on the coast of Esmeraldas. $X V I$ th International Congress of Americanists, vol. 2, Vienne, p. 331-345.

StiRLing M.W. (1963) - A new culture in Ecuador. Archaeology, New York, 16, p. 170-175.

Olivier DORIGHEL, Gérard POUPEAU et Erika LABRIN

Laboratoire de Géophysique Nucléaire Institut Dolomieu U.R.A. 69/C.N.R.S.

Université Joseph-Fourier 15, rue Maurice-Gignoux 38031 Grenoble

Jean-François BOUCHARD

U.P.T. 312 C.N.R.S., Archéologie de l'Amérique Préhispanique Musée de l'Homme Place du Trocadéro 75016 Paris 